\title{
Loeb Measures and Borel Algebras
}

\author{
H. Jerome Keisler and Yeneng Sun
}

\begin{abstract}
It is shown that a measurable function from an atomless Loeb probability space $(\Omega, \mathcal{A}, P)$ to a Polish space is at least continuum-to-one valued almost everywhere. It follows that there is no injective mapping $h:[0,1] \rightarrow \Omega$ such that $h([a, b])$ is Loeb measurable for each $0 \leq a<b \leq 1$ and $P(h([0,1]))>0$. Thus, when an atomless Loeb measurable algebra on an internal set of cardinality continuum is imposed on the unit interval $[0,1]$ through a bijection, it cannot contain the Borel algebra.

Keywords: Loeb measures, Lebesgue meassure, Borel algebras, bijections
\end{abstract}

\section{Introduction}

The Loeb measure construction introduced in [L] is a method from nonstandard analysis which is a valuable tool for proving standard results in mathematics (see, for example, [AFHL], [A] and [K1]). Recently, a number of special measure-theoretic properties of Loeb spaces were discovered and formulated in conventional terms. One is the neocompact approach developed in [FK1], [FK2], [K2] and [K3] which extracted the essential features of a nonstandard existence proof using Loeb spaces and provided a general procedure for going from approximate to limit. The others include a new theory of correspondences in [S2] and [S3] and a systematic study of the fundamental probabilistic concept of independence in [S1], [S4] and [S5] using Loeb product spaces.

A natural question which arises when the Loeb construction is used to obtain standard results is: what is a Loeb space? In applications one usually only needs to work with hyperfinite Loeb probability spaces in a nonstandard universe which is a countably indexed ultrapower. In that case, a hyperfinite set has the external cardinality of the continuum (the cardinality of the continuum will be denoted by c), so there is a bijection between the hyperfinite set and the unit interval $[0,1]$. Using this bijection, one can impose the Loeb measure structure on $[0,1]$. Thus, a Loeb measure can simply be viewed as a measure on the unit interval. In this setting, the special measure-theoretic properties of Loeb spaces can be restated as properties of some measure on the most familiar underlying space $[0,1]$.

Once a Loeb measure structure is imposed on the unit interval $[0,1]$ through a bijection, one may want to know its relationship with the Lebesgue measure structure. In particular, whether the imposed Loeb measurable algebra on $[0,1]$ 
could contain the Borel algebra, or more strongly, whether the imposed Loeb measure structure on $[0,1]$ could be an extention of the Lebesgue measure structure. An affirmative answer would alleviate the anxiety caused by unfamiliar notions in Loeb measure theory, such as internal sets, hyperfinite sets, or rich probability spaces. However, the results in this paper show that the answer to the above question is negative.

Note that if an imposed Loeb measurable algebra on $[0,1]$ contains the Borel algebra, then the relevant bijection from the original Loeb space must be Loeb measurable. Motivated by this observation, we consider the behavior of a general Loeb measurable mapping $f$ from an atomless Loeb probability space $(\Omega, \mathcal{A}, P)$ to a Polish space, and show that almost all points with respect to the image measure have at least continuum many preimages. Of course, this includes the particular case $X=[0,1]$. As a consequence, for any injective mapping $h:[0,1] \rightarrow \Omega$ in the other direction, if $h([a, b])$ is Loeb measurable for each $0 \leq a<b \leq 1$ then $P(h([0,1]))=0$. In the case that the mapping is a bijection, this shows that the answer to the previous question is negative.

For the convenience of the reader, the results are rigorously stated in Section 2 and the proofs are given in a separate Section 3. As usual, we work in an $\aleph_{1}$-saturated nonstandard universe (see [HL] for this basic definition).

\section{The results}

The following proposition shows that a measurable mapping $f$ from an atomless Loeb probability space to a Polish space $X$ is almost everywhere at least continuum-to-one valued.

Proposition 1 Let $f$ be a Loeb measurable mapping from an atomless Loeb probability space $(\Omega, \mathcal{A}, P)$ to a Polish space $X$ with distribution $\nu$ on $X$. Then, the inverse image set $f^{-1}(x)$ of $x$ has external cardinality at least the continuum for $\nu$-almost all $x \in X$.

The result cannot be improved by replacing " $\nu$-almost all $x \in X$ " by "all $x \in X$ ". In fact, starting from the above mapping $f$ and any $\nu$-null set $B$ in $X$, one can construct a Loeb measurable mapping $g$ with distribution $\nu$ such that for any $x \in B, g^{-1}(x)$ is at most a singleton. This mapping $g$ can be obtained by modifying the values of $f$ on the $P$-null set $f^{-1}(B)$.

Proposition 1 covers the case of a mapping from a Loeb space to $[0,1]$. In the other direction, we now consider injective mappings from $[0,1]$ to an atomless Loeb space. The next proposition shows that if the image set of each subinterval is Loeb measurable under such a mapping $h$, then the image of $h$ must be a $P$ null set.

Corollary 2 Let $(\Omega, \mathcal{A}, P)$ be a Loeb probability space, and let $h:[0,1] \rightarrow \Omega$ be an injective mapping such that $h([a, b])$ is Loeb measurable whenever $0 \leq a \leq$ $b \leq 1$. Then $P(h([0,1]))=0$. 
If our nonstandard universe is a countably indexed ultrapower, then any hyperfinite set has the same external cardinality as $[0,1]$. Thus, there are $2^{\mathbf{c}}$ many possible bijections between a hyperfinite set and $[0,1]$ in this case. Given any probability space $(\Omega, \mathcal{A}, P)$, any bijection $f$ from $\Omega$ to the unit interval $[0,1]$ imposes a probability space $([0,1], \mathcal{I}, \mu)$ on $[0,1]$ where

$$
\mathcal{I}=f(\mathcal{A})=\{f(A): A \in \mathcal{A}\}, \quad \mu(f(A))=P(A) .
$$

Let us say that such a probability space $([0,1], \mathcal{I}, \mu)$ is isomorphic to $(\Omega, \mathcal{A}, P)$. Given the abundance of such bijections, the result in the following corollary, which says that the induced isomorphic measure structures on $[0,1]$ can never contain the Borel structure, is somewhat surprising.

Corollary 3 Let $([0,1], \mathcal{I}, \mu)$ be a probability space which is isomorphic to an atomless Loeb probability space $(\Omega, \mathcal{A}, P)$. Then $\mathcal{I}$ cannot contain the Borel $\sigma$-algebra $\mathcal{B}$.

It is interesting to point out that given any atomless probability space $(\Omega, \mathcal{A}, P)$, if there is an isomorphic probability space on $[0,1]$ which contains the Borel algebra, then there is actually an isomorphic probability space on $[0,1]$ which is an extension of the Lebesgue measure structure. To see this, suppose that there is a probability space $([0,1], \mathcal{I}, \mu)$ isomorphic to $(\Omega, \mathcal{A}, P)$ through a bijection $f: \Omega \rightarrow[0,1]$ such that the $\sigma$-algebra $\mathcal{I}=f(\mathcal{A})$ contains the Borel algebra $\mathcal{B}$. Then, $\left([0,1], \mathcal{B},\left.\mu\right|_{\mathcal{B}}\right)$ is an atomless Borel probability space. The classical isomorphism theorem on Borel sets and Borel measures (see $[\mathrm{P}]$ ) implies the existence of a Borel isomorphism $h$ between the measure $\left.\mu\right|_{\mathcal{B}}$ and the Lebesgue measure $\lambda$ on $[0,1]$. Let $g$ be the composition of $f$ and $h$. Then, the measure structure on $[0,1]$ imposed by $g$ extends the Lebesgue measure structure.

\section{The proofs}

We begin with a lemma.

Lemma 4 Let $G$ be an internally measurable mapping from an atomless, internal probability space $(\Omega, \overline{\mathcal{A}}, \bar{P})$ to ${ }^{*} X$, where $X$ is a Polish space. Assume that $G(\Omega)$ is near standard in ${ }^{*} X$ for every $\omega \in \Omega$. Let $g(\omega)={ }^{\circ} G(\omega)$ for all $\omega \in \Omega$. Let $\mu$ be the distribution on $X$ induced by the mapping $g$ on the Loeb probability space $(\Omega, \mathcal{A}, P)$. Then, for any $x$ in the support of $\mu$, the external cardinality of $g^{-1}(x)$, the inverse image of the singleton $\{x\}$, is at least the continuum.

Proof Let $d$ be a metric on $X$ and $O_{n}$ the open ball $B(x, 1 / n)$ for every $n \geq 1$. Since $x$ is in the support of $\mu, \mu\left(O_{n}\right)=P\left(g^{-1}\left(O_{n}\right)\right)>0$. Thus, we can find an internally measurable subset $A_{n}$ of $\Omega$ such that $A_{n} \subseteq g^{-1}\left(O_{n}\right)$ with $\bar{P}\left(A_{n}\right)>\mu\left(O_{n}\right) / 2$. Hence, for any natural number $n \geq 1$,

(1) the internal cardinality $\left|A_{n}\right|$ of $A_{n}$ is greater than $n$, 
(2) $d\left(x, G\left(A_{n}\right)\right)<1 / n$.

By $\aleph_{1}$-saturation, the sequence $A_{n}, n \in \mathbf{N}$ can be extended to internally measurable subsets of $\Omega, A_{n}, n \in{ }^{*} \mathbf{N}$. By overspill, one can find an infinite $\gamma \in{ }^{*} \mathbf{N}$ such that for all $1 \leq n \leq \gamma$, Properties (1) and (2) hold. This means that $\left|A_{\gamma}\right|>\gamma$ and $d\left(x, G\left(A_{\gamma}\right)\right)<1 / \gamma$, which implies that $A_{\gamma} \subseteq g^{-1}(x)$. Hence the external cardinality of $g^{-1}(x)$ is at least the continuum.

\section{Proof of Proposition 1}

Let $F$ be an internal lifting of $f$. Take an increasing sequence of internally measurable sets $B_{n}$ in $\Omega$ such that $\bar{P}\left(B_{n}\right)>1-1 / 2^{n}$ and ${ }^{\circ} F(\omega)=f(\omega)$ for all $\omega \in B_{n}$.

Let $\bar{P}_{n}$ be the atomless, internal probability measure on $B_{n}$ defined by normalizing $\bar{P}$ restricted to $B_{n}$ and let $P_{n}$ be the relevant Loeb measure. Let $\mu_{n}$ be the distribution on $X$ induced by $\left.f\right|_{B_{n}}$. Then, Lemma 1 implies that for all $x$ in the support $S_{n}$ of $\mu_{n}$, the external cardinality of $\left(\left.f\right|_{B_{n}}\right)^{-1}(x)$ is at least the continuum.

Let $S=\cup_{n=1}^{\infty} S_{n}$. Then

$$
\begin{aligned}
\nu(S) \geq \nu\left(S_{n}\right) & =P\left(f^{-1}\left(S_{n}\right)\right) \geq P_{n}\left(\left(f_{B_{n}}\right)^{-1}\left(S_{n}\right)\right) \cdot P\left(B_{n}\right) \\
& =\mu_{n}\left(S_{n}\right) \cdot P\left(B_{n}\right)=P\left(B_{n}\right),
\end{aligned}
$$

which implies that $\nu(S)=1$. Hence, for all $x$ in $S$, the external cardinality of $f^{-1}(x)$ is at least the continuum.

\section{Proof of Corollary 2}

If there were such an $h$, and $f$ was the extension of $h^{-1}$ which sends the complement of $h([0,1])$ to 0 , then $f$ would be a Loeb measurable function with distribution $\nu$ such that the inverse image set $f^{-1}(x)$ is a singleton for any $x$ in the set $(0,1]$. But then

$$
\nu((0,1])=P\left(f^{-1}((0,1])\right)=P(h((0,1]))=P(h([0,1]))>0,
$$

which contradicts Proposition 1.

\section{Proof of Corollary 3}

Suppose that $\mathcal{I}$ does contain the Borel $\sigma$-algebra $\mathcal{B}$. Then the bijection $f$ is Loeb measurable. The distribution $\nu$ of $f$ is simply the restriction $\mu_{\mathcal{B}}$ of $\mu$ to $\mathcal{B}$. Proposition 1 implies that $f^{-1}(x)$ cannot be a singleton for $\nu$-almost all $x \in[0,1]$, which contradicts the bijective hypothesis on $f$.

The results were obtained while the authors were attending the Symposium on Reuniting the Antipodes - Constructive and Nonstandard Views of the Continuum at Venice, Italy on May 16-23, 1999. The authors are very grateful to Robert Anderson for helpful conversations and also to the organizers of the Venice conference, Ulrich Berger, Horst Osswald and Peter Schuster for the invitation to the conference. The research was supported in part by the National Science Foundation and the Vilas Trust Fund. 


\section{References}

[AFHL] S. Albeverio, J. E. Fenstad, R. Hoegh-Krohn, and T. L. Lindstrom, Nonstandard Methods in Stochastic Analysis and Mathematical Physics, Academic Press, Orlando, Florida, 1986.

[A] R. M. Anderson, A nonstandard representation for Brownian motion and Ito integration, Israel J. Math. 25 (1976) pp. 15-46.

[FK1] S. Fajardo and H. J. Keisler, Neometric spaces, Advances in Math. 118 (1996), pp. 134-175.

[FK2] S. Fajardo and H. J. Keisler, Existence theorems in probability theory, Advances in Math. 120 (1996), pp. 191-257.

[HL] A. E. Hurd and P. A. Loeb, An Introduction to Nonstandard Real Analysis, Academic Press, Orlando, Florida, 1985.

[K1] H. J. Keisler, An infinitesimal approach to stochastic analysis, Memoirs Amer. Math. Soc. 48 (1984), No. 297.

[K2] H. J. Keisler, Rich and Saturated Adapted Spaces, Advances in Math. 128 (1997), pp. 242-288.

[K3] H. J. Keisler, Quantifier elimination for neocompact sets, J. Symbolic Logic 63 (1998), pp. 1442-1472.

[L] P. A. Loeb, Conversion from nonstandard to standard measure spaces and applications in probability theory, Trans. Amer. Math. Soc. 211 (1975), pp. 113-122.

[P] K. R. Parthasarathy, Probability Measures on Metric Spaces, Academic Press, New York, 1967.

[S1] Y. N. Sun, Hyperfinite law of large numbers, Bull. Symbolic Logic 2 (1996), pp. 189-198.

[S2] Y. N. Sun, Distributional properties of correspondences on Loeb spaces, J. Functional Analysis 139 (1996), pp. 68-93.

[S3] Y. N. Sun, Integration of correspondences on Loeb spaces, Trans. Amer. Math. Soc. 349 (1997), pp. 129-153.

[S4] Y. N. Sun, A theory of hyperfinite processes: the complete removal of individual uncertainty via exact LLN, J. Math. Econ. 29 (1998), pp. 419-503.

[S5] Y. N. Sun, The almost equivalence of pairwise and mutual independence and the duality with exchangeability, Probability Theory and Related Fields 112 (1998), pp. 425-456. 
Department of Mathematics, University of Wisconsin-Madison 480 Lincoln Drive, Madison, Wisconsin 53706-1388, USA. keisler@math.wisc.edu Department of Mathematics, National University of Singapore

2 Science Drive 2, Singapore 117543, Republic of Singapore. matsuny@nus.edu.sg 